\title{
Higgs boson as a gluon trigger
}

\author{
P. Cipriano, ${ }^{1}$ S. Dooling, ${ }^{1}$ A. Grebenyuk, ${ }^{1}$ P. Gunnellini, ${ }^{1}$ F. Hautmann, ${ }^{2,3,4}$ H. Jung, ${ }^{1,5}$ and P. Katsas ${ }^{1}$ \\ ${ }^{1}$ Deutsches Elektronen Synchrotron, D-22603 Hamburg, Germany \\ ${ }^{2}$ Department of Physics and Astronomy, University of Sussex, Brighton BN1 9QH, United Kingdom \\ ${ }^{3}$ Physics Department, University of Oxford, Oxford OX1 3NP, United Kingdom \\ ${ }^{4}$ Rutherford Appleton Laboratory, Chilton OX11 OQX, United Kingdom \\ ${ }^{5}$ Elementaire Deeltjes Fysica, Universiteit Antwerpen, B-2020 Antwerp, Belgium
}

(Received 30 October 2013; published 26 November 2013)

\begin{abstract}
In the forthcoming high-luminosity phase at the LHC, many of the most interesting measurements for precision QCD studies are hampered by conditions of large pileup, particularly at not very high transverse momenta. We study observables based on measuring ratios of color-singlet currents via Higgs boson and Drell-Yan production, which may be accessed also at large pileup, and used for an experimental program on QCD physics of gluon fusion processes in the LHC high-luminosity runs. We present results of Monte Carlo calculations for a few specific examples.
\end{abstract}

DOI: 10.1103/PhysRevD.88.097501

PACS numbers: $14.80 . \mathrm{Bn}, 12.38 .-\mathrm{t}$

\section{INTRODUCTION}

The observation of the Higgs boson by the ATLAS and CMS experiments [1] marks the beginning of a revolutionary era in high-energy physics. It affects profoundly the paradigms by which we define the limits of our knowledge on the nature of interactions of elementary particles. This observation gives us confidence in the physical picture of fundamental interactions encoded by the Standard Model (SM) Lagrangian and provides us with guidance in the search for its generalizations.

The electroweak sector of the SM and the nature of electroweak symmetry breaking will be explored in detail in the coming years of operation of the LHC by measuring properties of the observed boson [2]. In this paper, we remark that the observation of the Higgs boson opens up the possibility of a rich experimental program in the strong-interaction sector of the SM as well. In particular, we propose that a program of QCD measurements at high luminosity can be carried out at the LHC by using the Higgs boson as a trigger, focusing on QCD gluonic processes at high mass scales.

Classic collider probes of QCD in $e^{+} e^{-}$annihilation, deep inelastic ep scattering, and Drell-Yan production (DY) all involve color-singlet currents which couple to quarks. With the Higgs, for the first time, LHC experiments will probe QCD by a color-singlet current which, in the heavy top limit, couples to gluons. The physics of gluon fusion processes can be explored from a new perspective compared to experimental investigations over the past three decades. As illustrated below, we propose measuring systematically differences of differential distributions for Higgs and Drell-Yan final states. This comparison allows one to access experimentally distinctive QCD features of gluon fusion physics.

In the next high-intensity phase at the LHC, one faces high pileup conditions leading to large numbers of overlaid events. In these conditions, many of the most interesting measurements for precision QCD studies, particularly for not very high transverse momenta, become extremely difficult-see e.g. Refs. [2,3]. Here we argue that by studying the differences of Higgs and Drell-Yan for masses around $125 \mathrm{GeV}$, the effects of pileup largely drop out. This offers the possibility of a program of QCD measurements of great physics interest in the high-luminosity runs of the LHC.

In this paper, we illustrate this by Monte Carlo simulation for three specific examples: the ratio of Higgs vs DrellYan $p_{\perp}$ spectra; the structure of the associated underlying event and charged-particle multiplicities; and the scattering angle in the center-of-mass reference frame. These involve QCD physics both at high transverse momenta and at low transverse momenta, and allow one to study both high- $x$ and low- $x$ physical effects.

We contrast the distinctive features of the Higgs trigger with other LHC short-distance probes such as jets, heavy flavors, and vector boson pairs which either couple perturbatively to color-octet and color-triplet sources on an equal footing, or imply final-state color-charged particles, or both.

We leave to detailed phenomenological investigations the study of the optimal channels to be used to access gluon fusion and suppress Higgs production by vector boson fusion and quark annihilation; of the luminosity requirements for reaching sufficient statistics; and of the different treatment of pileup for different channels.

Very recently, the ATLAS Collaboration has presented its first measurements of Higgs differential cross sections based on the 2012 data set in the diphoton decay channel [4].

\section{HIGGS VS DRELL-YAN}

Consider first transverse momentum spectra for Higgs bosons and for Drell-Yan (DY) pairs in the invariant mass range $115 \mathrm{GeV}<M<135 \mathrm{GeV}$. Transverse momentum spectra, comparing Higgs and $Z$ bosons, were examined 

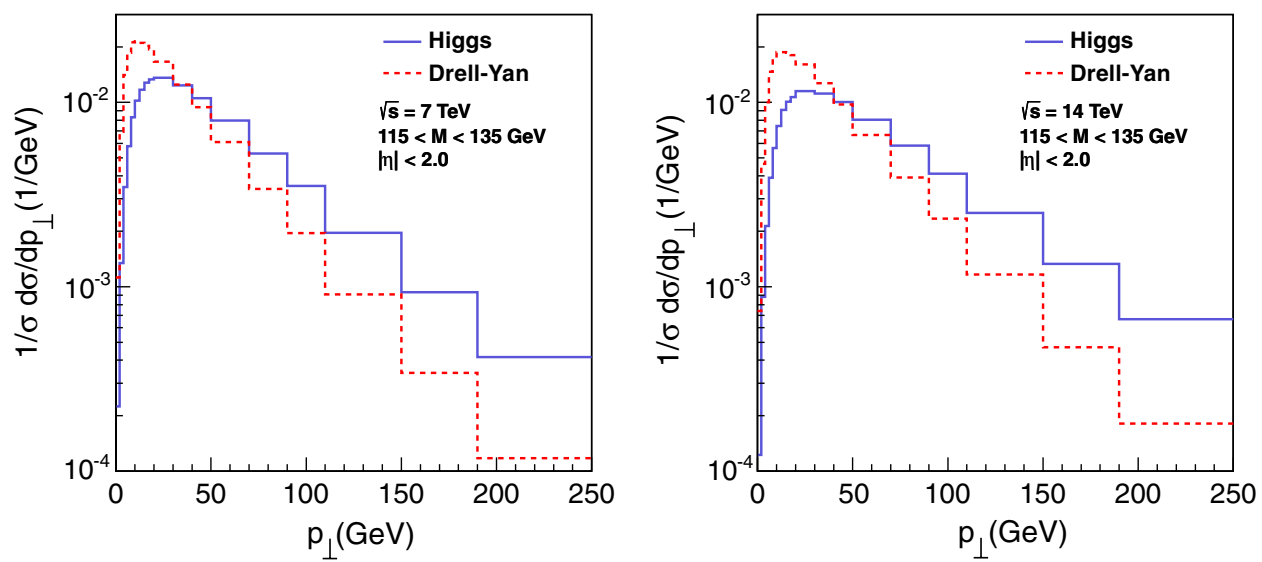

FIG. 1 (color online). Normalized transverse momentum spectra for Higgs bosons and for Drell-Yan pairs.

early on in Ref. [5]. The transverse momentum spectra can be described by QCD factorization in the form

$$
d \sigma / d p_{\perp}=\int H \otimes S \otimes J_{1} \otimes J_{2}
$$

decomposing the cross section into hard $(H)$, soft $(S)$, and collinear-to-initial-states $\left(J_{1}, J_{2}\right)$ contributions-see e.g. Ref. [6] for analysis of how this decomposition arises. In Fig. 1, we show the result of Monte Carlo simulations for the $p_{\perp}$ spectra in the central region based on the next-toleading-order (NLO) POWHEG [7] event generator interfaced with PYTHIA [8] shower, at $\sqrt{s}=7 \mathrm{TeV}$ and $\sqrt{s}=$ $14 \mathrm{TeV}$. In Fig. 2, we plot the ratio of the Higgs and DY spectra at invariant mass $115 \mathrm{GeV}<M<135 \mathrm{GeV}$.

The $p_{\perp} \ll M$ region of the spectrum measures infrared aspects of the cross section in Eq. (1); i.e., (i) the ratio of the gluon vs quark Sudakov form factor [factor $S$ in Eq. (1)], and (ii) the evolution of the collinear-to-initial-states functions [factors $J_{1}, J_{2}$ in Eq. (1)]. In particular, gluon polarization terms $p_{\perp}^{\mu} p_{\perp}^{\nu}$ in gluon fusion, related to eikonal polarizations at high energy [9], give rise to distinctive radiation patterns from initial-state functions in the Higgs case-see e.g. Ref. [10]. The $p_{\perp} \geq M$ region measures the ultraviolet function $H$ in Eq. (1) and the features of hard jets recoiling against the Higgs or DY pair. In particular, the leading-jet contribution to the measured ratio depends on the $p_{\perp}$ distribution for the spin- 1 vs spin- $1 / 2$ exchange and on the corresponding color emission probabilities. Further aspects on jet recoil are discussed below in the context of angular distributions.

In the large pileup environment of the high-luminosity LHC runs, one has to deal with the contribution of large numbers of overlaid events. However, this contribution cancels in the comparison of Higgs to DY spectra at fixed invariant mass. Using this comparison, one can go to low $p_{\perp}$ and access QCD effects in this region experimentally also at high pileup.

Measurements on gluon fusion which can be performed using the Higgs trigger open a new experimental area. They may also be relevant to interpreting data for other, more complex processes, e.g. processes that depend on both quark and gluon channels on an equal footing, or involve color-charged particles in the final state.

One such example is given by top quark production. This is often studied as a process sensitive to gluonic initial states at the LHC. For instance, the top quark $p_{\perp}$ spectrum [11] receives contributions at low $p_{\perp}$ from the gluon Sudakov form factor and gluonic initial-state recoil analogous to those discussed above. However, since the final
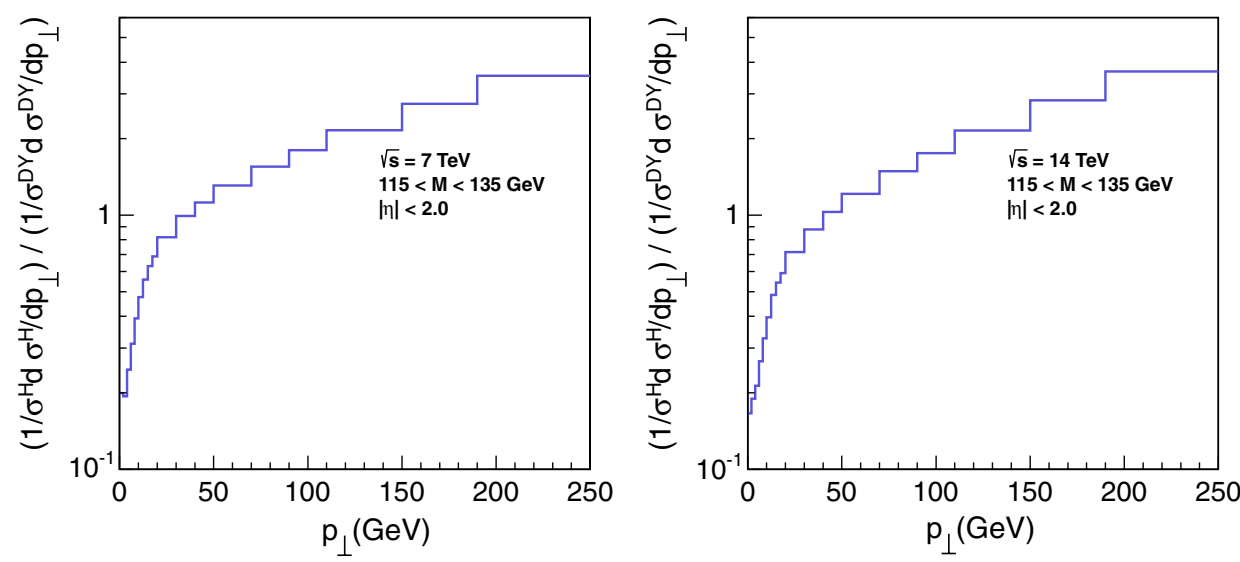

FIG. 2 (color online). Ratio of Higgs to DY spectra vs $p_{\perp}$. 
state is not a color-singlet current, the analysis of the $p_{\perp}$ spectrum is made more complex by final soft color emission. The Higgs case serves to single out the initial-state contributions, including gluon polarization effects.

For observables more exclusive than the cross section in Eq. (1), e.g. measuring the associated jets, full QCD factorization formulas are still lacking. For parton shower event generators, inclusive measurements are still useful to control methods [12,13] for merging parton showers and matrix elements. Higgs vs DY studies similar to those considered above can be done, for instance, in boson + jet states, now fixing, in addition to invariant mass, the jet transverse momentum or rapidity.

\section{UNDERLYING EVENTS}

The structure of underlying events and color flows associated with Higgs boson final states was investigated long ago [14] as a possible method to analyze $g g \rightarrow H$ and $W W \rightarrow H$ production mechanisms. In the case of vector boson final states it was pointed out [15] that the treatment of parton showers, and in particular of the recoils in the shower, is essential for a proper description of $W / Z$ spectra. This affects the amount of multiparton interactions [16] needed to describe the events $[15,17]$. Analogous effects may be investigated for gluonic showers $[18,19]$ in the case of events associated with Higgs final states.

We follow the treatment [20] of underlying events in the azimuthal plane, with the directions of the Higgs momentum and the DY-pair momentum, respectively, defining the origin in the azimuthal plane. In Figs. 3 and 4, we show the result of NLO POWHEG + PYTHIA Monte Carlo calculations for charged-particle multiplicities associated with Higgs and DY. (Analogous calculations can be usefully performed for multiplicities of minijets defined e.g. as in Ref. [21].) We plot the average multiplicity vs Higgs and DY $p_{\perp}$ (Fig. 3) and the multiplicity distribution (Fig. 4) in the transverse region of the azimuthal plane $\left(60^{\circ}<|\Delta \Phi|<120^{\circ}\right)$.

The distributions in the Higgs case are dominated by higher multiplicities from gluon cascades.
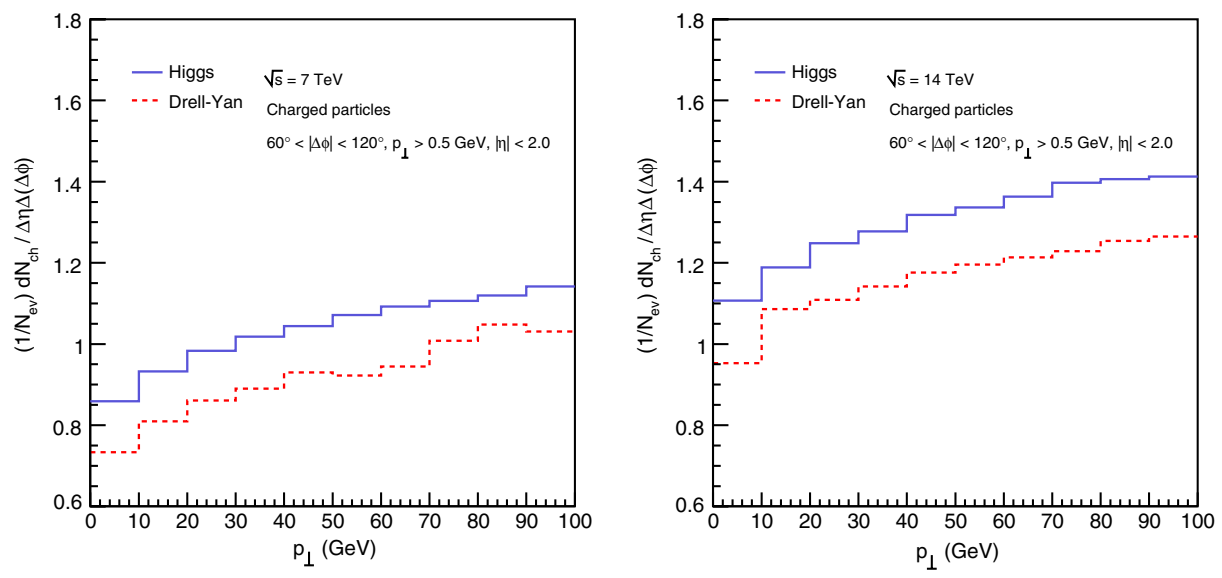

FIG. 3 (color online). Normalized charged-particle average multiplicity in the transverse region of the azimuthal plane vs the Higgs transverse momentum (solid blue line) and the DY transverse momentum (red dashed line).
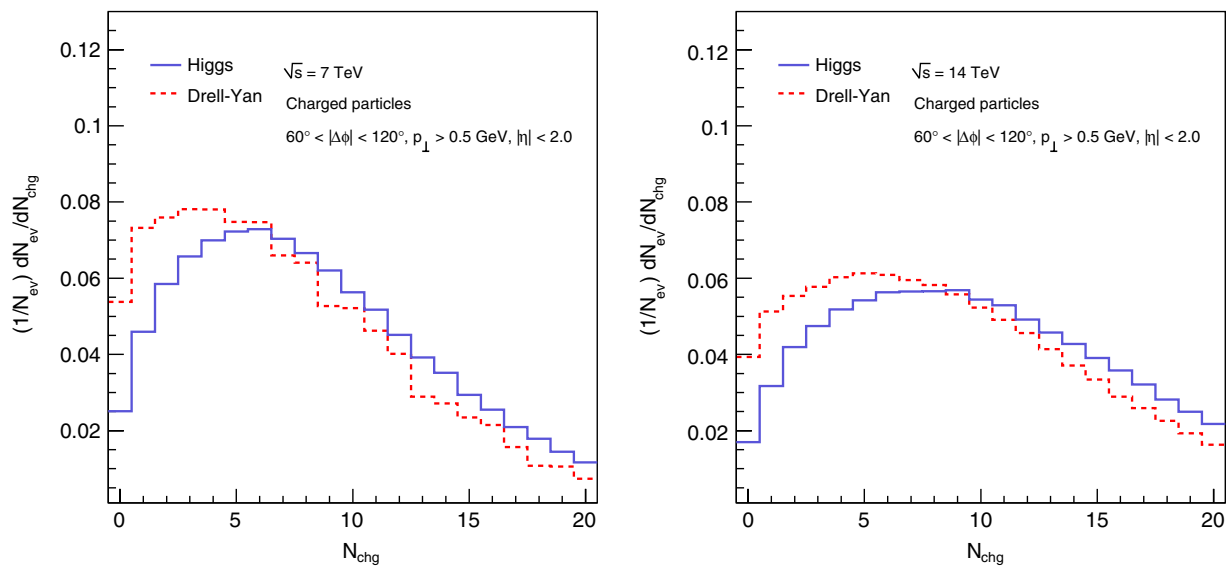

FIG. 4 (color online). Charged-particle multiplicity distribution in the transverse region of the azimuthal plane in the Higgs (solid blue line) and Drell-Yan (red dashed line) cases. 

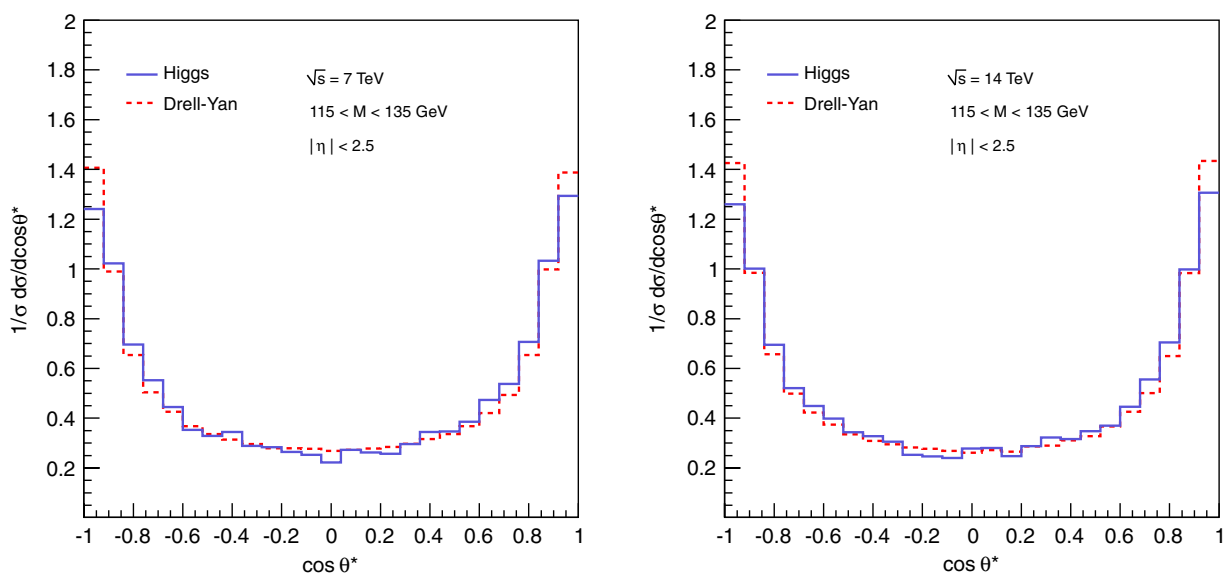

FIG. 5 (color online). Angular distribution in the center-of-mass scattering angle.

Similarly to the case of the previous section, the effects of a large number of overlaid events due to pileup will be reduced if one measures the difference between Higgs and DY underlying event distributions.

\section{ANGULAR DISTRIBUTIONS}

Besides soft radiation from underlying events, we consider Higgs vs DY distributions in the case of hard radiation accompanying the heavy bosons, for example boson + jet [22]. For Higgs production, the angular distribution in the scattering angle $\theta^{*}$ of the boson-jet center-ofmass frame is characterized by the scalar coupling to gluons partially canceling the small-angle Coulomb singularity $d \theta^{* 2} / \theta^{* 4}$ from gluon scattering-see e.g. Ref. [9]. The Drell-Yan $\theta^{*}$ distribution is determined by spin- $1 / 2$ exchange. Owing to the cancellation from the scalar coupling to gluons, the angular distributions have the same small-angle asymptotics in the Higgs and DY cases, despite the two processes occurring via spin- 1 and spin- $1 / 2$ exchange. The $\theta^{*} \rightarrow 0$ behavior thus tests the Higgs spin at the level of the production cross section.

In Fig. 5, we consider one-jet production associated with Higgs and $Z$ bosons, and show the differential distributions in $\cos \theta^{*}$, for jet $p_{\perp}>20 \mathrm{GeV}$ and boson-jet invariant mass $m$ such that $200 \mathrm{GeV}<m<500 \mathrm{GeV}$. The rise for increasing $\cos \theta^{*}$ reflects the mechanism described above. This large $\cos \theta^{*}$ power counting is the basic reason why the difference between Higgs and DY in the low- $p_{\perp}$ regions of Figs. 1 and 2 gives a measurement of higher-loop radiative contributions. Further effects from higher-order color emission may be analyzed via angular correlations in the boson-jet azimuthal plane in the laboratory frame.

In summary, this paper points out that a program of QCD measurements can be carried out in the high-luminosity phase at the LHC, using the Higgs boson as a gluon trigger. By measuring systematically differences between Higgs and Drell-Yan differential distributions for masses around $125 \mathrm{GeV}$, the effects of pileup largely cancel. Such measurements allow one to access experimentally, for the first time, gluon fusion processes at high mass scales via a colorsinglet current. Detailed studies are warranted to investigate quantitatively the reduction of pileup contributions in different channels, the optimal Higgs channels to access gluon fusion by suppressing vector boson fusion and quark annihilation, and the required Higgs statistics. The observables discussed in this paper illustrate that this program spans a broad range of physics issues on strong interactions, from soft gluon dynamics showing up in the ratio of Higgs to DY low- $p_{\perp}$ spectra, to underlying events and multiple parton interactions associated with gluonic showers, to hard-QCD contributions in large- $p_{\perp}$ spectra and angular distributions for boson + jet production. These angular distributions in particular may be used to test the spin of the Higgs at the level of production processes.

\section{ACKNOWLEDGMENTS}

F. H. gratefully acknowledges the hospitality and support of the Terascale Physics Helmholtz Alliance and DESY.
[1] G. Aad et al. (ATLAS Collaboration), Phys. Lett. B 716, 1 (2012); S. Chatrchyan et al. (CMS Collaboration), Phys. Lett. B 716, 30 (2012).

[2] ATLAS and CMS collaborations, submission to European Strategy for Particle Physics, Krakow, 2012.
[3] CMS Collaboration, "QCD at the Extremes", in European Strategy for Particle Physics, Krakow, 2012.

[4] ATLAS Collaboration, Report No. ATLAS-CONF-2013072.

[5] I. Hinchliffe and S. F. Novaes, Phys. Rev. D 38, 3475 (1988). 
[6] J. C. Collins and F. Hautmann, Phys. Lett. B 472, 129 (2000).

[7] S. Frixione, P. Nason, and C. Oleari, J. High Energy Phys. 11 (2007) 070.

[8] T. Sjöstrand, S. Mrenna, and P. Skands, J. High Energy Phys. 05 (2006) 026.

[9] F. Hautmann, Phys. Lett. B 535, 159 (2002).

[10] S. Mantry and F. Petriello, Phys. Rev. D 81, 093007 (2010); 83, 053007 (2011).

[11] CMS Collaboration, Report No. CMS-PAS-TOP-12-028; CMS Collaboration, Eur. Phys. J. C 73, 2339 (2013); ATLAS Collabortaion, Report No. ATLAS-CONF-2012-149.

[12] S. Plätzer, arXiv:1307.0774; J. High Energy Phys. 08 (2013) 114.

[13] L. Lönnblad and S. Prestel, Prog. Part. Nucl. Phys. 70, 1 (2013); arXiv:1211.7228.
[14] Yu. L. Dokshitzer, V. A. Khoze, and T. Sjöstrand, Phys. Lett. B 274, 116 (1992).

[15] P.Z. Skands, Phys. Rev. D 82, 074018 (2010).

[16] T. Sjöstrand and M. van Zijl, Phys. Rev. D 36, 2019 (1987).

[17] R. D. Field, arXiv:1010.3558.

[18] L. Lönnblad and M. Sjödahl, J. High Energy Phys. 02 (2004) 042; 05 (2005) 038.

[19] M. Deak et al., arXiv:1006.5401.

[20] S. Chatrchyan et al. (CMS Collaboration), J. High Energy Phys. 09 (2011) 109.

[21] M. Deak, F. Hautmann, H. Jung, and K. Kutak, Eur. Phys. J. C 72, 1982 (2012).

[22] R. K. Ellis, W. J. Stirling, and B. R. Webber, $Q C D$ and Collider Physics (Cambridge University Press, Cambridge, 1996). 